\title{
Service Robotics in Nursing Care. The Preliminary Results of a Scoping Review
}

\author{
Christoph OHNEBERG ${ }^{\mathrm{a}, 1}$, Nicole STÖBICH ${ }^{\mathrm{a}}$, Angelika WARMBEIN ${ }^{\mathrm{b}}$, Ivanka \\ RATHGEBER $^{\mathrm{b}}$, Uli FISCHER ${ }^{\mathrm{b}}$ and Inge EBERL ${ }^{\mathrm{a}}$ \\ ${ }^{a}$ Catholic University of Eichstätt-Ingolstadt, Germany \\ ${ }^{b}$ Hospital of the Ludwig-Maximilians-University (LMU) Munich, Germany
}

\begin{abstract}
A scoping review was prepared in the first study phase of the REsPonSe project. The objective was to gain an overview of existing literature and available evidence on the subject of service robotics in nursing care. The systematic literature search took place using the following databases: PubMed, CINAHL, Cochrane, Web of Science and IEEE Xplore. The titles and abstracts of 2.761 references were screened based on the inclusion criteria. A total of 31 articles were identified as relevant for the study.
\end{abstract}

Keywords: Assistive Robotics, Service Robots, Nursing Robotics, Scoping Review

\section{Introduction}

The aim of the REsPonSe project, which is sponsored by the Federal Ministry of Education and Research, is to alleviate the work of nursing care staff through the use of the Cliniserve ${ }^{\circledR}$ Smartphone-App combined with the autonomous service robot JEEVES ${ }^{\circledR}$, thereby providing them with more time for primary nursing tasks. Therefore, a scoping review [1] was prepared based on a systematic literature search. The objective was to gain an overview of existing literature and available evidence on the subject of service and assistant robotics in patient care. The aim was to identify the service and assistant robots used in ambulatory, residential and acute hospital settings, and to describe areas of application and the current state of development.

\section{Methods}

The systematic and iterative search was carried out in the following databases: PubMed, CINAHL, Cochrane, Web of Science and IEEE Xplore. An extended search took place using the lists of references in Google Scholar, CareLit, Springerlink and Pflege Hogrefe. The search was conducted from May to July 2020 without limiting the year of publication. The inclusion criteria were specified using the PCC formula (Population,

1 Corresponding Author Christoph Ohneberg, Catholic University of Eichstätt-Ingolstadt, Kapuzinergasse 2, 85072 Eichstätt-Ingolstadt, Germany; E-mail: Christoph.Ohneberg@ku.de. 
Concept, Context): a) Service/assistive robotics in the nursing care sector; b) Users: nursing care staff, service and assistant personnel, those in need of care, relatives; c) Language: English, German.

\section{Preliminary Results}

A total of 3.697 publications were found, 3.687 of which were located through the database search and a further ten through the extended search. Following the removal of duplicates, 2.761 articles were subjected to a blind screening of title and abstract by two reviewers. Of these, 87 publications qualified for the blind full text screening. Ultimately, 31 articles fulfilled the inclusion criteria and could be used for further analysis. The final selection of articles comprised eleven studies, ten technically related articles and nine texts and opinions. The publications originate from 14 different countries and were published during the period from 1998 to 2019. Appropriate assessment tools from the Joanna Briggs Institute were used to evaluate the studies.

\section{Discussion}

Robotic service systems are tested with user groups in pilot phases. The focus is mainly on usability and methods of development and application. The integration of robotic service systems in nursing care environments is barely examined past the test phase and the user perspective is seldom taken into account in the development of serviceoriented robotics $[2,3]$.

\section{Conclusion}

Intended users of robotic systems must be included in the development of application scenarios as well as in implementation. In order to increase user acceptance, researchers should closley monitor the pilot phase. The evaluation of future projects must include the perspective of nursing care staff. In addition, effects on nursing practice should also be assessed and the factors that support or impede implementation ascertained $[2,3,4]$.

\section{References}

[1] Tricco AC, Lillie E, Zarin W, O'Brien KK, Straus SE. PRISMA Extension for Scoping Reviews (PRISMA-ScR): Checklist and Explanation. Ann Intern Med. 2018;169(7):467-73.

[2] Mutlu B, Forlizzi J. Robots in Organizations: The Role of Workflow, Social, and Environmental Factors in Human-Robot Interaction. Carnegie Mellon University. 2008.

[3] Buhtz C, Paulicke D, Hirt J, Schwarz K, Stoevesandt D, Meyer G, Jahn P. Robotic systems for care at home: A scoping review. Zeitschrift für Evidenz Fortbildung und Qualität im Gesundheitswesen. 2018;137:1-8.

[4] Ienca M, Wangmo T, Jotterand F, Kressig RW, Elger B. Ethical Design of Intelligent Assistive Technologies for Dementia: A Descriptive Review. Sci Eng Ethics. 2018;24(4):1035-55. 